\title{
Retos de la Inclusión Académica de Personas con Discapacidad en una Universidad Pública Colombiana
}

\author{
Flavio H. Fernández-Morales y Julio E. Duarte \\ Universidad Pedagógica y Tecnológica de Colombia, Facultad Seccional Duitama, Grupo de Didáctica para \\ la Enseñanza de la Ciencia y la Tecnología en Niños, DECTEN, Carrera 18 Calle 23, Duitama-Colombia \\ (e-mail: flaviofm1@gmail.com; julioenriqued1@gmail.com)
}

Recibido Dic. 15, 2015; Aceptado Feb. 15, 2016; Versión final Feb. 26, 2016, Publicado Ago. 2016

\begin{abstract}
Resumen
Se presentan los resultados de un estudio que tuvo como objetivo el identificar las facilidades académicas e institucionales que brinda la Universidad Pedagógica y Tecnológica de Colombia a personas con discapacidad. La investigación fue de carácter exploratorio y se siguió una metodología de tipo descriptivo no experimental; se utilizaron la entrevista semi-estructurada y el cuestionario como herramientas para la recolección de información. Se entrevistó a estudiantes en situación de discapacidad, docentes y directivos, para identificar las políticas y planes con respecto a la inclusión; esto se complementó con el análisis de documentos como el estatuto general y algunos proyectos educativos. Los resultados evidencian el compromiso institucional con el cumplimiento de la normativa externa, relacionada con inclusión de discapacitados. Sin embargo, es notoria la falta de capacitación de los docentes en cuanto al manejo de estudiantes con necesidades educativas especiales, así como el desconocimiento de la comunidad académica con respecto a los derechos y posibilidades para la formación integral de discapacitados en la institución.
\end{abstract} integración.

\section{Challenges to Academic Inclusion of Disabled People at a Public Colombian University}

\begin{abstract}
The results of a study aimed to identify the academic and institutional facilities that the Universidad Pedagógica y Tecnológica de Colombia gives to disabled people. The research is of exploratory type and a follows a descriptive non-experimental methodology. A semi-structured interview and the questionnaire were used as tools for collecting information. Disabled students, professors, and executives were interviewed to identify policies and plans about inclusion. This was supplemented with analysis of documents such as the general statute and some educative projects. The results proved institutional commitment to fulfill external regulations relating to inclusion of disabled people. However, the lack of training of teachers is notorious about the handling of students with special educational needs, as well as the illiteracy of the academic community about rights and possibilities of integral formation of disabled people at the university.
\end{abstract}

Keywords: academic inclusion; disability; inclusive university; accessibility; support for integration. 


\section{INTRODUCCIÓN}

La forma como la sociedad trata a las personas con discapacidad ha evolucionado desde el modelo de la prescindencia, en el que se suponía que la discapacidad es de origen extra natural o divino, en el cual los discapacitados eran subvalorados hasta el punto de ser considerados innecesarios y la sociedad decidía no contar con ellos; un segundo modelo es el rehabilitador, en el que se busca determinar las causas científicas de la discapacidad, para desarrollar ayudas técnicas y tratamientos que permitan su rehabilitación; un tercer modelo es el llamado social, en el cual se considera que las causas que generan la discapacidad no son religiosas ni científicas sino sociales (Martín et al., 2013). En éste último modelo, la discapacidad se describe como una restricción o ausencia relacionada con una deficiencia de la capacidad para realizar cualquier actividad en lo que se conoce como normal para cualquier persona (Cáceres, 2004), citado por (Molina et al., 2011). En otras palabras, la discapacidad es aquella condición bajo la cual ciertas personas presentan alguna deficiencia sensorial, motora, intelectual o emocional que a largo plazo afectan la forma de interactuar y participar plenamente en la sociedad.

Recientemente se ha incrementado la vinculación de personas con discapacidad al sistema educativo, pasando del concepto de integración al de inclusión. Mientras que en la integración se trata de adaptar al alumnado a lo que hay, la inclusión directamente se ocupa de todos y cada uno; las personas con discapacidad no tienen por qué adaptarse a la normalidad, pues no son anormales, sino que el entorno y el medio les entorpecen su vivir (Padilla, 2011). La integración es un proceso continuo y progresivo, que inicia desde el grupo familiar, cuya finalidad es incorporar al individuo con necesidades especiales a la comunidad, y constituye uno de los fenómenos de mayor trascendencia en la educación (Romero y Lauretti, 2006). El movimiento inclusivo considera que los centros educativos deben satisfacer las necesidades de todos los alumnos, sean cuales sean sus características personales, psicológicas o sociales. La inclusión implica transformar la cultura, la organización y las prácticas de los centros para atender a la diversidad de necesidades educativas de todo el alumnado. Es una enseñanza adaptada al estudiante, cuyas acciones van dirigidas a eliminar o minimizar las barreras físicas, personales o institucionales que limitan las oportunidades de aprendizaje, el acceso y la participación en las actividades formativas (Álvarez et al., 2012).

Esta tendencia obedece a lineamientos de entidades multilaterales como la Organización de las Naciones Unidas, ONU, la Organización de las Naciones Unidas para la Educación, la Ciencia y la Cultura, UNESCO, y la Organización de Estados Iberoamericanos, OEl, entre otras, que propenden por la inclusión académica de personas con discapacidad, en especial al nivel de educación superior (Rodríguez y Álvarez, 2015; Hernández et al., 2015; Espinosa et al., 2012); esto debido a que los profesionales tienen mayor probabilidad de incidir en su entorno, mejorando las condiciones sociales y económicas de su contexto, rompiendo así con el ciclo de pobreza usualmente asociado a la discapacidad. En Colombia, partiendo de la Constitución Política, existe una gran cantidad de leyes, decretos y resoluciones, con normativa relacionada con la discapacidad y el derecho que asiste a las personas en esa condición a acceder a los servicios que brindan las instituciones de educación, en todos los niveles de formación (Hurtado y Agudelo, 2014; Molina, 2010).

En varios países Iberoamericanos algunos trabajos reportan avances relacionados con: la actitud de docentes y estudiantes hacia la inclusión académica de personas con discapacidad, formación del profesorado para atención de la discapacidad, diseños curriculares adecuados a la inclusión y políticas institucionales para atención a la discapacidad, entre otros (Novo et al., 2015; Moriña et al., 2013; Illanes y von Furstenberg, 2012; Ocampo, 2012). En Colombia también se han venido realizando esfuerzos para incorporar los propósitos de la inclusión educativa, especialmente en los niveles de educación preescolar, básica y media (Ossa, 2013). Sin embargo, para el caso colombiano, en las Instituciones de Educación Superior, IES, son escasos los estudios que permiten verificar los avances en materia de inclusión académica de personas con discapacidad. Las investigaciones en el área de educación superior, normalmente provienen de instituciones para personas con discapacidad y/o de las propias IES, interesadas en identificar el estado actual del proceso de inclusión, así como por estudiar estrategias para su implementación (Cruz et al., 2013).

La Universidad Pedagógica y Tecnológica de Colombia, UPTC, como institución pública de educación superior, tiene la obligación de desarrollar políticas de inclusión académica para las personas con discapacidad. Pese a lo anterior, en la actualidad los estudios acerca de la inclusión académica en la UPTC son escasos, lo cual limita la disponibilidad de reportes que permitan el desarrollo de estrategias sobre el tema (Medina y Niño, 2014). En éste trabajo se plantean los resultados de un estudio que buscó identificar las posibilidades que brinda la UPTC en aspectos académicos e institucionales, frente a la Inclusión Académica de Personas con Discapacidad, IAPD. En lo que resta del presente documento, se describen el tipo de estudio y la metodología, junto con la población e instrumentos de recolección de información. Luego 
se presentan los resultados, los cuales evidencian el compromiso institucional con el cumplimiento de la normatividad externa, relacionada con inclusión de personas en situación de discapacidad; sin embargo, es notoria la falta de capacitación de los docentes en cuanto al manejo de estudiantes con Necesidades Educativas Especiales, NEE. Finalmente se presentan las conclusiones, destacando la necesidad de sensibilizar a la comunidad académica con respecto a los derechos y posibilidades para la formación integral de personas en situación de discapacidad en la institución.

\section{METODOLOGÍA}

El objetivo general de la investigación fue: caracterizar los aspectos institucionales y académicos que permitan establecer el grado de inclusión que brinda la UPTC a la población con discapacidad. En este sentido, se propusieron como objetivos específicos los siguientes: identificar la normatividad y programas de bienestar universitario, existentes en la UPTC, para establecer el grado de inclusividad institucional; identificar los aspectos académicos, adecuaciones curriculares en los programas, capacitación de docentes y sensibilización de estudiantes, que facilitan la inclusión de personas en situación de discapacidad en la UPTC; establecer cuál ha sido la experiencia de estudiantes con discapacidad en su proceso formativo, para identificar las dificultades y fortalezas de la UPTC en cuanto inclusión académica.

La investigación fue de carácter exploratorio y se siguió una metodología de tipo descriptivo no experimental; se utilizaron la entrevista semi-estructurada y el cuestionario como herramientas para la recolección de información. En el primer semestre de 2015 la UPTC tenía 1674 docentes y contaba con 26.179 estudiantes matriculados, de los cuales 353 registraban algún tipo de discapacidad (UPTC, 2016). La población objeto de estudio estuvo conformada por 4 estudiantes con discapacidad, 33 docentes y 4 directivos de la UPTC, cuya muestra se seleccionó por conveniencia y quienes aceptaron participar de forma voluntaria en el estudio, previo consentimiento informado. Se entrevistaron 4 estudiantes de sexo masculino, cuyas edades varían entre 17 y 26 años, con las siguientes características: E1, estudiante de Licenciatura en ciencias sociales, con discapacidad motriz; E2, estudiante de Licenciatura en música, con discapacidad visual de baja visión; E3, estudiante de Ingeniería electrónica, con discapacidad motriz y E4, estudiante de Contaduría, con una alteración en la médula espinal que periódicamente le genera discapacidad visual y motriz. Las entrevistas fueron grabadas para su análisis. Las siguientes fueron las preguntas planteadas a los estudiantes:

P1: ¿Considera que la UPTC es una institución inclusiva?, ¿Por qué?

P2: ¿Ha sido beneficiario de algún programa de la Unidad de Política Social, UPS?

P3: ¿La UPTC dispone de los espacios, material adaptado y asesorías, requeridos para su formación?

P4: ¿En su opinión, los docentes están capacitados para desarrollar las adaptaciones curriculares requeridas?

P5: ¿Cómo considera la comunicación con sus compañeros?

P6: ¿Cuál considera que es la actitud de los demás estudiantes hacia los personas en situación de discapacidad?

P7: ¿Cómo considera la comunicación con sus docentes?

P8: ¿Cuál considera que es la actitud de los docentes hacia los personas en situación de discapacidad?

En total se consultaron 33 docentes, de los cuales 18 pertenecen a diversos programas, 8 corresponden al programa de Psicopedagogía, 2 son directores de Ingeniería de sistemas e Ingeniería geológica, mientras que los 5 restantes son directores de los programas de licenciatura en: Educación Preescolar, Psicopedagogía, Educación Industrial, Matemáticas y Estadística y Educación Física. Las siguientes fueron las preguntas planteadas a los docentes:

Q1: ¿Ha participado en eventos relacionados con Inclusión Académica de Personas con Discapacidad, IAPD?

Q2: ¿Conoce la normativa de la UPTC relacionada con la IAPD?

Q3: ¿Cuál es su actitud frente a estudiantes con discapacidad?

Q4: ¿Ha recibido capacitación para el manejo del proceso de enseñanza aprendizaje en personas con discapacidad?

Q5: ¿Ha trabajado con estudiantes en situación de discapacidad en la UPTC?

Q6: ¿Ha utilizado adaptaciones curriculares para personas en situación de discapacidad en su área de desempeño?

Q7: ¿Conoce algún programa de la UPS para el apoyo a personas con discapacidad?

También se contactó a los siguientes directivos, seleccionados por su relación directa con la formulación, desarrollo, ejecución y evaluación de políticas institucionales sobre discapacidad: vicerrector académico, 
director de la unidad de política social, director de bienestar universitario y jefe de registro. Lo anterior se complementó con el análisis de documentos como el estatuto general y los Planes Académicos Educativos, PAE, de 6 programas, a saber: Psicopedagogía, Educación Física, Matemáticas y Estadística, Preescolar, Licenciatura en Educación Industrial e Ingeniería de Sistemas.

\section{RESULTADOS Y DISCUSIÓN}

La tabla 1 presenta la opinión de los estudiantes con discapacidad, frente a su proceso formativo en la UPTC, cuyas respuestas permiten una aproximación a su experiencia personal y académica en la institución. La primera columna indica el número de la pregunta, cuyo enunciado se mencionó en la sección anterior; además, $\mathrm{S}$ significa sí, $\mathrm{N}$ no, mientras que $\mathrm{B}$ significa bueno, $\mathrm{R}$ regular y $\mathrm{M}$ malo.

Tabla 1: Respuestas de estudiantes con discapacidad

\begin{tabular}{|l|l|l|l|l|l|l|l|l|l|l|l|l|l|l|l|l|l|l|l|l|}
\multicolumn{1}{l|}{} & E1 & \multicolumn{10}{l|}{ E2 } & \multicolumn{10}{l|}{ E3 } \\
\hline P & S & N & B & R & M & S & N & B & R & M & S & N & B & R & M & S & N & B & R & M \\
\hline P1 & X & & & & & X & & & & & & X & & & & & X & & & \\
\hline P2 & X & & & & & X & & & & & & X & & & & & X & & & \\
\hline P3 & & X & & & & X & & & & & & X & & & & & X & & & \\
\hline P4 & & X & & & & & X & & & & & X & & & & X & & & & \\
\hline P5 & & & X & & & & & X & & & & & X & & & & & & X & \\
\hline P6 & & & X & & & & & & X & & & & & X & & & & & X & \\
\hline P7 & & & X & & & & & & & X & & & X & & & & & X & & \\
\hline P8 & & & X & & & & & & X & & & & & X & & & & & X & \\
\hline
\end{tabular}

A P1, dos de los estudiantes manifiestan que la UPTC si es una institución inclusiva ya que permite el ingreso a los programas y las instalaciones de la sede central tienen una accesibilidad aceptable; sin embargo, perciben deficiencias en el acompañamiento académico durante su proceso formativo. E3 y E4 consideran que la UPTC no es inclusiva ya que en la sede en que ellos se encuentran, los edificios no cuentan con rampas ni señalización, impidiendo el acceso a servicios básicos como los de la Unidad de Política Social, UPS. Se destaca que los estudiantes consideran la accesibilidad de las instalaciones como un referente de la inclusión institucional, y su percepción varía dependiendo de la Facultad en que se encuentren. Así, quienes utilizan los edificios históricos, con más de 10 años de antigüedad, tienen problemas para su desplazamiento, pues cuando fueron construidos no existían normas arquitectónicas que tuvieran en cuenta la accesibilidad como un requerimiento de diseño.

A P2, dos de los estudiantes han sido beneficiarios del programa de becas de la UPS, mientras que los otros dos no, argumentando desconocimiento de las posibilidades que brinda la institución a éste respecto. Actualmente ninguno de los entrevistados está inscrito en ningún programa deportivo o cultural, aduciendo falta de información por parte de la UPS, lo cual limita su formación integral. A P3 solo un estudiante manifestó haber empleado material Braille en una de sus asignaturas, mientras que los demás desconocen la existencia de materiales o servicios de asesoría que faciliten su proceso formativo. Ante P4, solo uno de los estudiantes manifiesta que sus docentes han desarrollado las adaptaciones curriculares para su integración en el aula. Los otros tres estudiantes manifiestan no haber empleado adaptaciones curriculares e incluso detectan desconocimiento y nerviosismo por parte de sus docentes, al no saber cómo actuar con ellos. A P5, tres de los estudiantes consideran que la relación con sus compañeros es buena, percibiendo en ellos aceptación y colaboración. E4 manifiesta una buena comunicación con sus compañeros, pero percibe falta de solidaridad de su parte en lo que respecta a la movilidad.

A P6, en cuanto a la actitud de los demás estudiantes frente a los personas en situación de discapacidad, solo un estudiante considera que es buena; los otros tres, aunque no se han sentido discriminados, consideran que podría existir una mayor cultura a la hora de permitir el desplazamiento en silla de ruedas y/o con el bastón, dar espacio; igualmente recomiendan implementar una política de igualdad, para evitar que los demás consideren a los personas en situación de discapacidad como inútiles o incapaces de rendir académicamente. Esto último se complementa con lo expresado por el encargado de la UPS: "Miren que es curioso lo que encontramos en la población con limitaciones físicas: ellos nos ayudan a descubrir que quienes más necesitan de formación inclusiva somos los que, entre paréntesis, nos consideramos supuestamente normales; porque no los sabemos entender a ellos". 
A P7, comunicación con sus docentes, tres de los estudiantes consideran tener una buena comunicación con sus docentes y haber recibido apoyo de su parte, mientras que solo E2 indica lo contrario. En éste último caso, el problema radica en que le exigen trabajos manuscritos y la lectura de gran cantidad de material impreso, lo cual es bastante difícil por el poco residuo visual que aún mantiene. A P8, solo un estudiante manifiesta una buena actitud por parte de los docentes hacia los personas en situación de discapacidad, mientras que los demás consideran que es regular aunque sin discriminación; ellos perciben cierto nerviosismo e incomodidad de su parte, al no saber cómo actuar ante la situación, en especial cuando tienen el primer contacto.

La tabla 2 presenta la opinión de los docentes frente a su experiencia en el manejo de la discapacidad, cuyas respuestas permiten una aproximación a la actitud personal e institucional en cuanto a inclusión de estudiantes con discapacidad se refiere. La primera columna indica el número de la pregunta, cuyo enunciado se mencionó en la sección anterior; en esta tabla, para las respuestas se utilizó la misma nomenclatura de la tabla 1.

Tabla 2: Respuestas de los docentes entrevistados

\begin{tabular}{|l|l|l|l|l|l|l|l|l|l|l|l|l|l|l|l|}
\cline { 2 - 17 } \multicolumn{1}{c|}{ DOCENTES } & \multicolumn{4}{l|}{ PSICOPEDAGOGÍA } & \multicolumn{3}{|c|}{ DIRECTORES } \\
\hline Preguntas & $\mathrm{Si}$ & No & $\mathrm{B}$ & $\mathrm{R}$ & $\mathrm{M}$ & $\mathrm{Si}$ & No & $\mathrm{B}$ & $\mathrm{R}$ & $\mathrm{M}$ & $\mathrm{Si}$ & No & $\mathrm{B}$ & $\mathrm{R}$ & $\mathrm{M}$ \\
\hline Q1 & 3 & 15 & & & & 7 & 1 & & & & 6 & 1 & & & \\
\hline Q2 & 1 & 17 & & & & 5 & 3 & & & & 4 & 3 & & & \\
\hline Q3 & & & 12 & 6 & & & & 7 & 1 & & & & 7 & & \\
\hline Q4 & 3 & 15 & & & & 3 & 5 & & & & 3 & 4 & & & \\
\hline Q5 & 10 & 8 & & & & 3 & 5 & & & & 6 & 1 & & & \\
\hline Q6 & 4 & 14 & & & & 1 & 7 & & & & 4 & 3 & & & \\
\hline Q7 & 3 & 15 & & & & 6 & 2 & & & & 2 & 5 & & & \\
\hline
\end{tabular}

A Q1, 16 docentes han participado en eventos relacionados con IAPD, mientras que los 17 restantes no lo han hecho. Es interesante observar que la mayoría de directores de escuela y docentes de Psicopedagogía han participado en eventos académicos relacionados con inclusión y discapacidad; en el caso de los primeros, esto se debe a la responsabilidad de su cargo que los obliga a conocer la normativa relacionada con la temática; mientras que para los segundos, la temática de inclusión hace parte de su ejercicio profesional. A Q2, solo 10 docentes conocen la normativa institucional relacionada con discapacidad, mientras que los restantes 23 no la conocen. Al igual que en Q1, la mayoría de directores conoce la normativa institucional, así como los docentes de psicopedagogía, quienes incluso han participado en la formulación de políticas relacionadas con IAPD.

A Q3, pregunta relacionada con la actitud frente a estudiantes con discapacidad, 26 docentes manifiestan tener buena disposición, mientras que los 7 restantes indican que su actitud es regular. En general los docentes presentan una buena actitud frente a estudiantes con discapacidad, resaltando el derecho que les asiste a formarse a nivel superior; algunos mencionan la incomodidad que les podría generar la situación de tener un discapacitado en el aula, en especial por la falta de conocimientos para lograr su inclusión. A Q4, 24 de los entrevistados no han recibido capacitación para el manejo de estudiantes con discapacidad, mientras que los restantes 9 si lo han hecho, pero antes de laborar como docentes de la UPTC. A Q5, 14 de los docentes no han tenido la experiencia de trabajar en el aula con estudiantes personas en situación de discapacidad, mientras que los restantes 19 si lo han hecho. Este último grupo destaca que es una experiencia enriquecedora, que genera relaciones de igualdad y respeto en el aula.

A Q6, 24 docentes no han utilizado adaptaciones curriculares para estudiantes con discapacidad, mientras que solo 9 manifiestan haberlas empleado pero en instituciones diferentes a la UPTC. Estas respuestas son coherentes con Q4, indicando que la falta de capacitación limita el buen desempeño docente, en especial en temas relacionados con una adecuada atención a las Necesidades Educativas Especiales, NEE. A Q7, 22 de los entrevistados no conocen ningún programa de la UPS para discapacitados, mientras que los restantes 11 si los conocen. Vale la pena destacar que la mayoría de los docentes de Psicopedagogía han participado en el desarrollo de programas para personas en situación de discapacidad, los cuales se constituyen en escenario de práctica para sus estudiantes.

Al revisar el Estatuto General de la UPTC, en su Artículo 88, se indica: "La universidad reconoce que los miembros de la comunidad universitaria con discapacidades físicas, permanentes o temporales, sensoriales, 
cognitivas, y necesidades especiales, requieren protecciones especiales, de manera que estas no incidan en las condiciones de equidad académica o laboral; por tanto, se establecerán políticas tendientes a garantizar el desarrollo de sus potencialidades humanas, físicas y académicas." (UPTC, 2005, p. 27).

Es interesante observar que la UPTC cuenta en su normativa con lineamientos en los que se reconoce a las personas con discapacidad como parte integrante de la comunidad universitaria, indicando la necesidad de establecer una política institucional que favorezca la inclusión de personas con discapacidad. Además, los directivos manifiestan el conocimiento pleno de la normativa gubernamental sobre inclusión de estudiantes con discapacidad, la cual es de obligatorio cumplimiento por parte de las instituciones de educación superior.

Sin embargo, aunque se han elaborado algunas propuestas como lo manifestaron los directivos entrevistados y los docentes en Q2, a la fecha no se cuenta con ningún Acuerdo o documento oficial que reglamente la política de inclusión; esto último constituye un gran inconveniente, pues la falta de normativa explícita sobre inclusión de estudiantes con discapacidad, limita la disponibilidad de información, dificulta la difusión de la temática y el desarrollo de las políticas requeridas para que la UPTC sea una institución realmente inclusiva.

Al no existir una política oficial de inclusión, los encargados de Registro Académico no han podido generar una base de datos relacionada con los estudiantes personas en situación de discapacidad; esto a su vez limita la disponibilidad de información oportuna y veraz, que permita trazar las políticas y programas relacionados con la inclusión. La falta de normativa también limita el servicio de las instancias administrativas, ya que los funcionarios no disponen de procedimientos e información que les permitan brindar una atención adecuada a los estudiantes con discapacidad. Consecuencia de ello es que, como lo manifestaron en P2, dos de los estudiantes entrevistados no han recibido orientación con respecto a las posibilidades que brinda la UPTC para favorecer su proceso formativo. En éste sentido se advierte la necesidad de incrementar los esfuerzos de sensibilización a la comunidad universitaria, a través de foros y conferencias que pongan de manifiesto los derechos de la población con discapacidad, creando así una verdadera cultura de inclusión dentro de la institución.

En la UPTC, la Unidad de Política Social, UPS, es la dependencia que apoya las funciones misionales de la institución, propendiendo por la formación integral de la comunidad y una equitativa distribución de las oportunidades y beneficios que posibiliten el desarrollo humano (UPTC, 2015). Esta función se cumple a través de la oficina de Bienestar Universitario, la cual prevé brindar un servicio incluyente, que contemple situaciones de discapacidad y acceso de grupos vulnerables, como minorías étnicas y desplazados, entre otros (UPTC, 2014). En éste sentido se proveen servicios de salud, Psicología, actividades deportivas y culturales, y se cuenta con programas de becas que benefician a toda la comunidad educativa. Sin embargo, como lo manifiestan los directores de la UPS y de Bienestar Universitario, no existen programas dirigidos específicamente a la población con discapacidad, sino que éstos se enmascaran en los servicios previstos para la población vulnerable, en la que se incluyen: extrema pobreza, desplazados por el conflicto interno y minorías étnicas. La falta de actividades deportivas y culturales específicamente diseñadas para los personas en situación de discapacidad, unida a la escasa información que se brinda a estudiantes y docentes, se traducen en una casi nula vinculación de discapacitados en los grupos universitarios.

Según los expertos hablar de inclusión no solo es hablar de planes de estudio, también se deben evidenciar espacios culturales y deportivos en donde se integre a toda la comunidad universitaria sin importar ninguna diferencia o condición (Bernal, 2013). Este es un aspecto que la UPTC deberá considerar en su política de atención a la discapacidad, estableciendo planes que garanticen el acceso de los personas en situación de discapacidad a una verdadera formación integral.

Los Planes Académicos Educativos, PAE, en la UPTC tienen como propósito orientar y definir las políticas de desarrollo académico de cada programa; por ello se constituyen en insumo importante para identificar los aspectos académicos y adecuaciones curriculares relacionados con la inclusión. En los PAE analizados se observa una preocupación por el respeto a los derechos humanos, en los términos establecidos en la Constitución Política y en las normas del Ministerio de Educación Nacional, MEN; además se habla de retomar dentro de la escuela aspectos que modifiquen y mejoren el currículo, en bien de la sociedad y la comunidad educativa. Sin embargo, en las mallas curriculares es notoria la ausencia de asignaturas que preparen a los egresados en temáticas relacionadas con inclusión y diversidad.

Se esperaría que, especialmente, en los programas de licenciatura, donde se forman los futuros docentes, se trabajaran las NEE, las adaptaciones curriculares y el tratamiento a la diversidad, aspectos fundamentales de la práctica docente. Lo anterior cobra relevancia ya que el MEN (2014), recientemente expidió los lineamientos de calidad para las licenciaturas en educación, donde se reglamenta la existencia de condiciones de accesibilidad para estudiantes con limitaciones, exigiendo estrategias y actividades para 
el desarrollo de programas de educación inclusiva en cualquier programa de formación de docentes. Una excepción la constituye el programa de Psicopedagogía, el cual evidencia asignaturas que destacan la importancia de plantear propuestas para incluir la diversidad como una realidad educativa; en su perfil profesional se indica la capacidad de realizar diagnósticos, identificar y analizar situaciones en diferentes contextos para proponer adaptaciones curriculares dirigidas a población con NEE, promoviendo los procesos de inclusión educativa y social (UPTC, 2009).

En cuanto a los aspectos académicos relacionados con la IAPD, como: lineamientos pedagógicos, metodológicos y adaptaciones curriculares, los PAE brindan poca orientación. Los Directores de programa indicaron que lo relacionado con el tema era más un desarrollo por interés propio, que la aplicación de algún procedimiento establecido institucionalmente. Esto se traduce en que los casos de discapacidad se tratan puntualmente; es decir, cuando un estudiante con discapacidad ingresa a un programa, se hace una valoración de su situación y se intenta buscar apoyo en dependencias como Bienestar universitario. En éste sentido, sería deseable que las mallas curriculares contemplen asignaturas que permitan reflexionar sobre las implicaciones de la inclusión, promoviendo así un cambio en la mentalidad de estudiantes y docentes frente al tema.

Otro aspecto que limita las posibilidades de inclusión académica de estudiantes con discapacidad en la UPTC es la falta de capacitación de los docentes. Esta situación se evidencia en los PAE, donde no se contempla la capacitación docente como fundamento para la inclusión. Además, en P4 y P8, los estudiantes observan poca experticia por parte de los docentes para su inclusión en el aula, lo cual impide el uso de adecuaciones curriculares que beneficien su proceso formativo. Si bien la actitud de los docentes frente a la inclusión es positiva, la falta de capacitación en el desarrollo y aplicación de adecuaciones curriculares se constituye en un obstáculo para la inclusión, ya que son los responsables de elaborar y llevar al aula los lineamientos establecidos en las mallas curriculares.

Para que una universidad sea inclusiva es necesario que sus docentes también lo sean. No basta con buenas intenciones, es necesario que los docentes reciban una formación que les permita, con base en un diagnóstico, establecer las estrategias pedagógicas, metodológicas y didácticas, que garanticen una adecuada atención a la discapacidad (Fernández et al., 2015); así será posible cumplir con uno de los postulados de la inclusión académica: procurar los mecanismos para el ingreso, seguimiento, desarrollo y egreso de los estudiantes con discapacidad, de modo que puedan vincularse de forma productiva a su contexto.

Adicionalmente, es necesario sensibilizar a estudiantes, docentes y demás miembros de la comunidad universitaria, sobre los derechos y posibilidades que brinda la UPTC para la inclusión de personas con discapacidad, así como sobre el derecho que asiste a éste colectivo a beneficiarse de medidas que garanticen su acceso a la institución, su ingreso en los centros, su permanencia en la universidad y el ejercicio de sus derechos académicos en igualdad de condiciones y sin discriminación alguna.

\section{CONCLUSIONES}

La inclusión académica es un mecanismo para asegurar que los estudiantes en situación de discapacidad tengan la posibilidad de acceder a todos los recursos de una institución educativa, sin ninguna discriminación. Los resultados, obtenidos en la institución analizada, evidencian el compromiso institucional con el cumplimiento de la normatividad externa, relacionada con inclusión de personas en situación de discapacidad; sin embargo, es notoria la falta de capacitación de los docentes en cuanto al manejo de estudiantes con Necesidades Educativas Especiales, NEE, así como el desconocimiento de la comunidad académica con respecto a los derechos y posibilidades para la formación integral de personas en situación de discapacidad en la institución.

Entre las fortalezas, se tiene el reconocimiento institucional del derecho de los estudiantes con discapacidad a recibir una educación de calidad, en condiciones de equidad; esto se une a la buena disposición de la comunidad universitaria para formar una institución incluyente.

Sin embargo, es imprescindible establecer una política clara que favorezca la inclusión, que contemple planes y programas como: establecimiento de estrategias pedagógicas orientadas a la atención de las NEE, capacitación docente en el uso de adaptaciones curriculares, desarrollo de programas culturales y deportivos que coadyuven en la formación integral de los estudiantes con discapacidad, todo lo anterior unido a estrategias de comunicación y sensibilización que permitan la formación o el fortalecimiento de una comunidad universitaria que acoja a los personas en situación de discapacidad sin discriminación alguna. 


\section{AGRADECIMIENTOS}

Los autores agradecen a la Dirección de Investigaciones de la UPTC, por la financiación del proyecto con código SGI-1764. Igualmente agradecen a Carlos Medina y Freddy Niño, Licenciados en Educación Industrial de la UPTC, por su colaboración en la recolección de la información.

\section{REFERENCIAS}

Álvarez, P., O. Alegre y D. López, Las dificultades de adaptación a la enseñanza universitaria de los estudiantes con discapacidad: un análisis desde un enfoque de orientación inclusiva, doi: 10.7203/relieve.18.2.1986, Relieve, 18(2), art. 3, (2012)

Bernal, C. A., Aplicación de sistemas de clasificación en contextos educativos: Facilitando los procesos de inclusión de personas en situación de discapacidad intelectual, rev.fac.med., ISSN: 0120-0011(en línea; http://www.scielo.org.co/scielo.php?script=sci_arttext\&pid=S0120-00112013000200004\&lng=es\&nrm=iso.

Acceso: 6 de agosto 2015), 61(2), 20-25, (2013)

Cruz, I., C. Duarte, A. Fernández y S. García, Caracterización de investigaciones en discapacidad en Colombia 2005-2012, rev.fac.med ISSN: 2357-3848 (en línea; http://www.revistas.unal.edu.co/index.php/revfacmed/article/view/39641/47346, Acceso: 16 de julio 2015) 61(2), 101-109, (2013)

Espinosa, Carlos X, Gómez, Víctor G, \& Cañedo, Carlos M., El Acceso y la Retención en la Educación Superior de Estudiantes con Discapacidad en Ecuador, doi: 10.4067/S0718-50062012000600004, Form.Univ (en línea), 5(6), 27-38 (2012)

Fernández, F. H., J. E. Duarte y G. J. Gutiérrez, Estrategia pedagógica para la formación de ingenieros con discapacidad visual, Pap. Trab. - Cent. Estud. Interdiscip. Etnolinguist. Antropol. Soc., ISSN: 1852-4508 (en línea; http://www.scielo.org.ar/scielo.php?script=sci_arttext\&pid=S185245082015000100003\&lng=es\&nrm=iso, Acceso: 2 de septiembre 2015), 29, 36-48,(2015)

Hernández, C., Márquez, H. y Martínez, F., Propuesta Tecnológica para el Mejoramiento de la Educación y la Inclusión Social en los Niños Sordos, doi: 10.4067/S0718-50062015000600013, Form. Univ. (en línea) 8, (6), 107-120 (2015)

Hurtado, L. D. y A. Agudelo, Inclusión educativa de las personas con discapacidad en Colombia, Revista ces Movimiento y Salud, ISSN: 2357-562X (en línea;

http://revistas.ces.edu.co/index.php/movimientoysalud/article/view/2971. Acceso: 7 de julio 2015), 2(1), 45-

55, (2014)

Illanes, L. y M. T. von Furstenberg, Implementación de un programa de inclusión a la educación superior de jóvenes con necesidades educativas especiales por discapacidad cognitiva en la Universidad Andrés Bello, doi: 10.4151/07189729-Vol.51-Iss.2-Art.111, Perspectiva Educacional (en línea), 51(2), 69-87 (2012)

Martín, E., P. Sarmiento y L. Coy, Educación inclusiva y diversidad funcional en la Universidad, Rev. Fac. Med., 61(2), 195-204 (2013)

Medina, C. y F. Niño, La inclusión académica de estudiantes con discapacidad de la UPTC, Tesis de Pregrado, Universidad Pedagógica y Tecnológica de Colombia, Facultad Seccional Duitama, Colombia, (2014)

MEN, Ministerio de Educación Nacional, Lineamientos de calidad para las licenciaturas en educación, Viceministerio de Educación Superior, (en línea; http://www.mineducacion.gov.co/cvn/1665/articles340962_recurso_1.pdf. Acceso: 18 de agosto 2014), Colombia, (2014)

Molina, D., F. De Bedoya y Y. Sánchez, Integración de los Estudiantes con Discapacidad en las Aulas universitarias, Rev. Mex. Orient. Educ., ISSN 1665-7527 (en línea;

http://pepsic.bvsalud.org/scielo.php?script=sci_arttext\&pid=S1665-75272011000100005\&lng=pt\&nrm=iso.

Acceso: 7 de junio 2015), 8(20), 33-44, (2011)

Molina, R. M., Educación superior para estudiantes con discapacidad, Revista de investigación, ISSN: 10102914, (en línea; http://www.scielo.org.ve/scielo.php?script=sci_arttext\&pid=S101029142010000200008\&lng=es\&nrm=iso. Acceso: 2 de agosto 2014) 34(70), 95-115, (2010) 
Moriña, A., R. López, N. Melero, M. Cortés y V. Molina, El profesorado en la universidad ante el alumnado con discapacidad: ¿tendiendo puentes o levantando muros?, REDU, ISSN: 1887-4592,(en línea; http://www.red-u.net/, Acceso: 13 de mayo de 2015),11(3), 423-442, (2013)

Novo, I., J. Muñoz y N. Calvo, Los futuros docentes y su actitud hacia la inclusión de personas con discapacidad: una perspectiva de género, doi: 10.6018/analesps.31.1.163631, Anal. Psicol., 31(1), 155-171 (2015)

Ocampo, A., Inclusión de estudiantes en situación de discapacidad a la educación superior: desafíos y oportunidades, Revista Latinoamericana de Inclusión Educativa, ISSN-e: 0718-7378 (en línea; http://www.rinace.net/rlei/numeros/vol6-num2/art10.pdf. Acceso: 13 de febrero (2015), 6(2), 227-239, (2012)

Ossa, C., Actitudes de estudiantes sobre personas con discapacidad en la Universidad del Bío-Bío, Psicogente, ISSN-e: 2027-212X (en línea;

http://portal.unisimonbolivar.edu.co:82/rdigital/psicogente/index.php/psicogente, Acceso: 23 de enero 2015), 16(29), 32-42 (2013).

Padilla, A., Inclusión educativa de personas con discapacidad, rev. colomb. psiquiatr., ISSN: 0034-7450 (en línea; http://www.scielo.org.co/scielo.php?script=sci_arttext\&pid=S0034-

74502011000400007\&lng=en\&nrm=iso. Acceso: 25 de noviembre 2014), 40(4), 670-699, (2011)

Rodríguez, A. y E. Álvarez, Universidad y discapacidad: Actitudes del profesorado y de estudiantes, Perfiles educativos, ISSN: 0185-2698 (en línea; http://www.scielo.org.mx/scielo.php?script=sci_arttext\&pid=S018526982015000100006\&lng=es\&nrm=iso, Acceso: 25 de agosto 2015), 37(147), 86-102, (2015)

Romero, R. y P. Lauretti, Integración educativa de las personas con discapacidad en Latinoamérica, Educere, ISSN: 1316-4910 (en línea; http://www.scielo.org.ve/scielo.php?script=sci_arttext\&pid=S131649102006000200019\&lng=es\&nrm=iso. Acceso: 9 de diciembre 2014), 10(33), 347-356, (2006)

UPTC, Universidad Pedagógica y Tecnológica de Colombia, Acuerdo 066/2005: Estatuto General de la UPTC, Consejo Superior (en la web: http: www.uptc.edu.co-export-sites-default-secretaria_generalconsejo_superior-acuerdos_2005-Acuerdo_066_2005.pdf, Acceso: 9 de octubre 2014) Colombia, (2005)

UPTC, Universidad Pedagógica y Tecnológica de Colombia, Modificación al plan maestro institucional 2007 - 2019, (en la web: http--www.uptc.edu.co-export-sites-default-universidad-documentos-plan_maestroplan_maestro.pdf. Acceso: 9 de abril 2015) Colombia, (2014)

UPTC, Universidad Pedagógica y Tecnológica de Colombia, RESOLUCIÓN 062/2009: Reestructuración del plan de estudios del programa de licenciatura en psicopedagogía con énfasis en asesoría educativa de la UPTC, Consejo Académico, (en la web: http--www.uptc.edu.co-export-sites-default-secretaria_generalconsejo_academico-resoluciones_2009-res_62_2009.pdf, Acceso: 8 de marzo 2015) Colombia, (2009)

UPTC, Universidad Pedagógica y Tecnológica de Colombia, Informe cuatrienio 2011 - 2014 UPTC, (en línea: http--www.uptc.edu.co-export-sites-default-universidad-acerca_de-inf_comunidad-doc-2015inf_cuatrienio_2011_2014.pdf, Acceso: 19 de abril 2015) Colombia, (2015)

UPTC, Universidad Pedagógica y Tecnológica de Colombia, Rendición de cuentas vigencia 2015 (en la web:http://www.uptc.edu.co/export/sites/default/universidad/acerca_de/rendicion_cuentas/2016/doc/informe _rcuentas2015.pdf, acceso 24 de febrero de 2016), Colombia, (2016) 
